\title{
Ayda Ignez Arruda e Sua Trajetória Profissional e Científica na UNICAMP (1968-1983)
}

\author{
Suélen Rita Andrade Machado \\ Faculdade de Engenharia e Inovação Técnico Profissional - FEITEP - Brasil \\ Lucieli M. Trivizoli \\ Universidade Estadual de Maringá - UEM - Brasil \\ (aceito para publicação em outubro de 2021)
}

\begin{abstract}
Resumo
Neste artigo nosso objetivo é caracterizar historicamente a trajetória profissional e científica da professora catarinense Ayda Ignez Arruda na Universidade Estadual de Campinas (Unicamp), no período de 1968 a 1983. Os dados que aqui apresentamos correspondem a resultados de uma pesquisa de doutorado que está sendo desenvolvida no âmbito do Programa de Pós-Graduação em Educação para a Ciência e a Matemática da Universidade Estadual de Maringá. A pesquisa tem por objetivo a composição de um estudo históricobiográfico de Ayda Ignez Arruda e suas contribuições para a institucionalização da lógica matemática no Brasil. Para a escrita deste artigo, utilizamos a abordagem qualitativa e os pressupostos da escrita biográfica, na construção de uma narrativa que conduz vestígios históricos documentais e bibliográficos sobre a personagem. Baseadas nessa interface, evidenciamos que Ayda Ignez Arruda foi professora titular na área de Lógica e Fundamentos da Matemática Instituto de Matemática, Estatística e Computação Científica da Unicamp, participou do Grupo de Lógica de Campinas, fez intercâmbios e visitas a outras instituições, organizou eventos e seminários, foi membro fundadora do Centro de Lógica e Epistemologia (CLE), além de ser sido diretora do IMECC e membro fundadora da Sociedade Brasileira de Lógica (SBL).
\end{abstract}

Palavras-chave: Matemática, História, Ayda Ignez Arruda, Lógica Matemática. 


\title{
[Ayda Ignez ARruda AND Her Professional AND SCIENTIFIC History at UniCAMP (1968-1983)]
}

\begin{abstract}
In this article, our objective is to historically characterize the professional and scientific path of the professor Ayda Ignez Arruda from Santa Catarina at the State University of Campinas (Unicamp), from 1968 to 1983. This paper presents the data from a a $\mathrm{PhD}$ research developed on the Graduate Program in Education for Science and Mathematics at the State University of Maringá. That research aims to compose a historical-biographical study by Ayda Ignez Arruda and her contributions to the institutionalization of Mathematical Logic in Brazil. Writing this paper, we have chosen a qualitative approach and the assumptions of biographical writing, for the construction of a narrative that leads to historical documentary and bibliographic traces about the person. Based on this line, we verified that Ayda Ignez Arruda was a full professor in the field of Logic and Fundamentals of Mathematics at the Institute of Mathematics, Statistics and Scientific Computing (IMECC) of Unicamp, she participated in the Campinas Group of Logic, she exchanges and visits to other institutions, organized conferences and seminars, she was a founding member of the Center for Logic and Epistemology (CLE), in addition to being the director of IMECC and a founding member of the Brazilian Society of Logic (SBL).
\end{abstract}

Keywords: Mathematics, History, Ayda Ignez Arruda, Mathematical Logic.

\section{Introdução}

Dentre as possibilidades investigativas em história da matemática no Brasil se identificam os estudos histórico-biográficos sobre personas que desempenharam ações em prol da institucionalização e desenvolvimento da matemática no país. Essas pesquisas (BADIN, 2006; SOUTO, 2006; PEREIRA, 2010; CAVALARI, 2012; BONFIM, 2013; LEMOS, 2013; COBRA, 2014; DUARTE, 2014; CALABRIA, 2015; ARAUJO NETO, 2019) tem demonstrado que personagens singelos ${ }^{1}$ (por não estarem em constantes holofotes) tiveram seus nomes marcados na raiz acadêmica de sociedades e instituições. Também tem mostrado que esses(as) personagens foram notáveis e decisivos(as) na história do desenvolvimento e institucionalização de diversos campos da Matemática no Brasil.

A partir das pesquisas que situamos anteriormente, conjecturamos uma incipiente presença feminina em termos de estudos histórico-biográficos. Entendemos que esse é um desdobramento da predominância masculina na história, relegando as mulheres das ciências. De acordo com Lopes (2014) até meados do século XIX, universidades e sociedades científicas recém-criadas não previam a participação feminina. Foi necessário um longo caminho para que as mulheres pudessem ser reconhecidas em direito na produção do

${ }^{1}$ Alusão a Araújo Neto (2019). 
conhecimento científico. E ainda, as mulheres "[...] foram "escondidas da história", ou seja, sistematicamente excluídas da maioria dos relatos dos historiadores" (JENKINS, 2007, p. 26).

Contudo, esse pesquisador revela que há pessoas engajadas no reconhecimento de grupos omissos da história, por entender a necessidade de revelar a participação dessas pessoas que foram importantes para a sociedade. Nesse viés nos identificamos, pois entendemos a necessidade da pesquisa histórica revelar a participação masculina, mas também a participação feminina nos mais diversos campos da matemática. Face a isso, em nossa pesquisa de doutorado desenvolvida no âmbito do curso de pós-graduação em Educação para a Ciência e Matemática da Universidade Estadual de Maringá, compomos um estudo histórico-biográfica sobre a professora catarinense Ayda Ignez Arruda e suas constribuições para a institucionalização da lógica matemática no Brasil. Neste artigo, com a apresentação de resultados parciais da referida pesquisa, nossa intenção é caracterizar historicamente a trajetória profissional e científica de professora catarinese Ayda Ignez Arruda na Universidade Estadual de Campinas (Unicamp), nos período de 1968 a 1983.

Para tanto, nos valemos do entrelaçamento documental e bibliográfico sob a forma de uma narrativa biográfica que leva em consideração os pressupostos analíticos denominados por Trivizoli (2016) de zooming in e zooming out. Focando em detalhes específicos do que se deseja analisar e escrever, em nosso caso, detalhes sobre a trajetória científica e profissional de Ayda na Unicamp - zooming in; e olhando para uma perspectiva mais ampla sobre o objeto, ou seja, outras situações que permeavam a vida de Ayda zooming out. A narrativa em uma biografia, segundo Delory-Momberger (2012, p. 525) é uma "[...] forma de discurso que mantém a relação mais direta com a dimensão temporal da existência e da experiência humana".

E ainda, quando tratamos de narrativa histórica, afeta a vestígios documentais, vislumbramos uma convergência em uma tendência historiográfica contemporânea que, de acordo com Saito (2015) e Trivizoli (2016), exibe diversas fontes documentais de diferentes campos do conhecimento (não se limitando apenas à matemática). Desse modo: “[...] fazem parte desse conjunto de documentos não só livros e tratados, mas também cartas, manuscritos, minutas e outros documentos não só escritos, mas também aqueles da cultura material, tais como instrumentos, monumentos, máquinas etc" (SAITO, 2015, p. 27) o que possibilita o entendimento amplo e não isolado do objeto a qual se investiga.

Os vestígios documentais primários que dispomos sobre Ayda Arruda foram obtidos a partir do Fundo AIA $^{2}$ do Centro de Lógica, Epistemologia e História da Ciência (CLE) Unicamp, do Arquivo Central do Sistema de Arquivos (SIARQ) - Unicamp, da Hemeroteca Digital Brasileira, a partir de jornais da época e arquivos de familiares. Os vestígios documentais secundários foram obtidos a partir de artigos, teses e dissertações. Além da proximidade à análise documental para a escrita deste artigo, levamos em conta que a escrita biográfica leva em consideração a flexibilidade e o não engessamento metodológico da escrita (BORGES, 2002). Assim, os dados que consideramos significativos e são trazidos aqui, resultam do acesso e disponibilidade das fontes que dispomos.

\footnotetext{
${ }^{2}$ Sigla referente ao nome Ayda Ignez Arruda.
} 
De fato, entendemos que pesquisa biográfica é aquela que permite capturar e escrever cientificamente uma parte da história de um determinado(a) personagem, levando em consideração os vestígios do passado que tornam possível tal construção, bem como o estabelecimento de constantes e permanências em relação ao espaço temporal, social etc. Ademais, são traços de uma trajetória que pode ser enfatizada por vieses: seja pessoal, profissional ou acadêmica, neste artigo enfatizada pelo viés profissional e científico.

Assim, para este artigo apresentaremos parte da história de Ayda Ignez Arruda, enfatizando sua vivência profissional e científica na Unicamp, nos anos de 1968 até 1983. Para tanto, primeiramente apresentaremos Ayda e sua ascendência acadêmica, brevemente alguns aspectos históricos de sua trajetória no Paraná, posteriomente, sua ida a CampinasSP, seu engajamento e o fomento de sua carreira. Por fim, algumas considerações serão tecidas.

\section{Aspectos históricos da trajetória de vida de Ayda: SC/PR.}

Ayda Ignez Arruda, nasceu no dia 27 de junho de 1936 no distrito de Painel-SC, filha de Lourenço Waltrick Arruda e Isabel Pereira do Amarante. Em 1945, se mudou para Lages-SC junto à sua família, e lá cursou o primário no "Colégio Santa Rosa de Lima" - 1947 (Na época Internato e Externato dirigido pelas irmãs da Providência ${ }^{3}$ ), o curso ginasial no "Colégio Diocesano" - 1951 e o curso Normal na "Escola Normal Vidal Ramos" - 1954, concluindo, também nessa cidade, sua educação secundária. Para dar início aos seus estudos superiores, mudou-se para Curitiba-PR, cursou o curso pré-vestibular na época, sendo aprovada em segundo lugar no curso de matemática da Pontifícia Universidade Católica do Paraná. Assim, bacharelou-se em matemática no ano de 1958 e licenciou-se em 1959 neste curso e nesta mesma instituição.

Em 1957, no segundo ano do seu curso de matemática, Ayda conheceu o professor Newton Carneiro Affonso da Costa, que havia assumido uma Cadeira na instituição na qual Ayda estudava. Newton da Costa foi discípulo do português João Remy Teixeira Freire, personagem catalisador para a Matemática no Paraná e criador da Sociedade Paranaense de Matemática (SPM) e descendente científico de Bento de Jesus Caraça, também criador da Sociedade Portugesa de Matemática (SPM) (ARAUJO NETO, 2019). Nessa época, o professor Newton da Costa começou a desenvolver uma série de seminários sobre Lógica e Fundamentos da Matemática, e Ayda além de participar destes seminários, começou a se interessar pelos estudos desenvolvidos por aquele que seria seu futuro orientador.

No ambiente de Curitiba, berço do desenvolvimento da lógica no Brasil, trabalhou na Universidade Federal do Paraná e na Universidade Católica do Paraná, nos anos 1960 a 1968, instituições nas quais estabeleceu elos acadêmicos com diversos personagens, dentre eles o professor Newton Carneiro Affonso da Costa como já elencamos, pioneiro da lógica matemática no Brasil, com quem exerceu importantes papéis na Sociedade Paranaense de Matemática - SPM, compondo o grupo que ficou conhecido como Escola de Curitiba.

Marcel Guillaume (2007) da Universidade de Clermont-Ferrand, França, citado por Gomes e D’Ottaviano (2017) destacou que quando passou o verão de 1964-1965 (durante

${ }^{3}$ (ESTADO DE SANTA CATHARINA, 1930, p. 1006). 
três meses) em Curitiba-PR, como convidado do professor Newton para ser professor na Universidade Federal do Paraná, participou dos seminários de Lógica de seu amigo Newton, afirmando que eram momentos bastante produtivos, com a presença de no máximo três pessoas: ele (Guillaume), Newton e a jovem Ayda Arruda, como ele mesmo cita. Ainda elenca que o progresso do estudo das lógicas paraconsistentes $C_{n}$ fez sucesso nesse tempo.

De acordo com Aliseda e Manzano (2015) foi nesse momento que Marcel Guillaume, Newton da Costa e Ayda Arruda, ainda aluna deste último elaboraram diversos trabalhos que introduziram uma hierarquia de conjuntos paraconsistentes. Ainda em 1964, Ayda começou a publicar seus primeiros trabalhos em colaboração com Newton da Costa (D'OTTAVIANO; CARNIELli; ALVES, 1996). Contudo, Ayda já tinha publicações datadas de 1963 e já era notável o papel de importância que adquiria na construção das lógicas paraconsistentes junto a da Costa:

"[...] Ela foi a primeira a trabalhar no projeto teórico e nos sistemas paraconsistentes de da Costa, antes mesmo da publicação dos primeiros resultados. Ayda Arruda, conforme seu relato e o de Mário Tourasse, já trabalhava nas lógicas paraconsistentes $C_{n}$ desde o seu desenvolvimento inicial, participando dos seminários, debates, estudos conjuntos e dirigidos conduzidos por Newton da Costa em Curitiba, no célebre Grupo de Álgebra e Lógica." (GOMES; D'OTTAVIANO, 2017, p. 421, grifo nosso)

Ainda segundo esses autores, Ayda Ignez Arruda era muito estudiosa e foi colaboradora de Newton desde o início. Ayda começou sua carreira profissional ainda na década de 1960 e, em 1966, obteve o título de doutora e livre-docente com a tese Considerações sobre os Sistemas Formais $N F_{n}$, produzida sob a orientação do professor da Costa.

De fato, a pesquisa original em Lógica no Brasil, começou com o trabalho pioneiro do professor Newton da Costa na cidade de Curitiba, capital do Estado do Paraná: "[...] Sob a liderança de da Costa realizaram-se seminários e, a partir de 1957, constituiu-se um pequeno grupo de lógica, devendo ser salientada a participação de Ayda Ignez Arruda, a primeira discípula e colaboradora de da Costa" (D'OTTAVIANO; GOMES, 2011b, p. 7, grifo nosso). Além de primeira discípula e colaboradora de Newton, conjecturamos que ela também foi uma das primeiras na área no país, enquanto mulher.

Deste contexto inferimos que dois eventos foram significativos tanto para a Lógica quanto para o desenvolvimento da Matemática no Paraná, a criação da Sociedade Paranaense de Matemática - SPM, ${ }^{4}$ em 1953 e a fundação do Instituto de Matemática da Universidade do Paraná - IMUP, em 1959. A SPM teve o professor português João Remy Teixeira Freire como responsável por sua criação e promotor de parcerias importantes de professores filiados, como já elencamos. Realizou seminários, reuniões, conferências, publicação de livros, periódicos (no Anuário e no Boletim da Sociedade Paranaense de Matemática -

${ }^{4}$ Desde 2002, a sede da SPM foi transferida para Universidade Estadual de Maringá - UEM.

RBHM, Vol. 21, n 41, pp. 25-44, 2021 
BSPM $^{5}$ ), que se estabeleciam como meios de divulgação e trocas de publicações de sociedades, universidades brasileiras e do exterior (COUSIN, 2007; SANTOS; MIYAÒKA; BARREDA, 2016; ARAUJO NETO, 2019).

Por outro lado, no ano de 1958, têm-se o marco inicial da pesquisa em Matemática na UFPR com a fundação do Centro de Ensino e Pesquisas de Matemática e Estatística, vinculado ao Instituto de Matemática da Universidade do Paraná - IMUP. Por meio do registro no livro de Atas vê-se nomes de personagens relacionados ao Conselho TécnicoCientífico: Zélia Milléo Pavão, Newton Carneiro Affonso da Costa, Hugo Frederico Kremer, Léo Barsotti e Jayme Machado Cardoso) Entre os anos 1958-1959 foram promovidos pelo Centro, seminários e cursos de extensão ministrados por professores de São Paulo e Rio de Janeiro, com compras de livros e revistas especializadas em Matemática e Estatística, com concessão de bolsas etc. (SANTOS; MIYAÒKA; BARREDA, 2016).

Da Costa e Sant'Anna (2016) destacam que o ambiente de Curitiba de certo modo foi influenciado pelo desenvolvimento matemático que ocorria na Polônia, que nos anos 1919-1929 despontou no cenário internacional da Matemática mesmo de modo incipiente em relação às grandes potências com dedicação a Lógica, a Topologia, Fundamentos da Matemática e Análise Funcional. Apesar da pobre realidade local, Newton da Costa presenciou esse apogeu quando da sua passagem pela Universidade de Torun, na Polônia.

Desse modo, sob influência desse despontar, da Costa tentou reproduzir em Curitiba o mesmo ambiente hegemônico matemático polonês, em parceria com a Escola de Curitiba e/ou Grupo de Curitiba, composto por Jayme Machado Cardoso, Haroldo C. A. da Costa, Ayda Ignez Arruda, Zélia M. Pavão, Iromi Inoue, Nathan M. dos Santos, Antonio Santa Rose e Darcy Modesto. Da Costa planejou e implementou “[...] um Seminário de Lógica e Álgebra assim que ingressou no Instituto de Matemática. Seu propósito inicial era formar uma massa crítica de interessados em lógicas não clássicas e teoria dos reticulados" (DA COSTA; SANT'ANNA, 2016, p. 99). Como fruto da dedicação desse grupo, fomenta-se no Brasil, os estudos relacionados a lógica matemática, a partir da criação das lógicas paraconsistentes e da teoria dos reticulados de Cury.

Nesse contexto, ainda no IMUP, destaca-se a visita de Elon Lages de Lima, Maria Laura Mouzinho, Constantino Menezes de Barros, Artibano Micali e o norte-americano Marshall Stone. Ademais, outra visita importante na década de 1960 foi a do francês Marcel Guillaume, que contribuiu para a matemática nesse momento histórico e influenciou o perfil profissional de da Costa e de Jayme Machado Cardoso. Guillaume teve papel decisivo no nascimento e no desenvolvimento da lógica brasileira (DA COSTA; SANT'ANNA, 2016). Ainda sobre a Escola de Curitiba podemos destacar que em pouco tempo:

“[...] o Grupo de Curitiba ampliou seus propósitos para o estudo de filosofia da ciência, com especial destaque em matemática e física. Isso porque tanto a lógica quanto a álgebra naturalmente se inserem em inúmeras áreas das ciências formais (como a matemática) e reais (especialmente a física) e demandam o estudo de muitas disciplinas correlatas." (DA COSTA; SANT'ANNA, 2016, p. 100)

\footnotetext{
${ }^{5}$ O Boletim da Sociedade Paranaense de Matemática - BSPM foi fundado em 1953. O BSPM é o mais antigo jornal de Matemática Brasileiro impresso, tem publicado durante esse período, importantes trabalhos de pesquisa de Matemática e de Lógica do mundo todo. Disponível em: 〈http://www.spm.uem.br/_bspm/index.php〉.
} 
De acordo com Da Costa e Alcântara (1985, p. 2, tradução nossa), os jovens lógicos e matemáticos integrantes desse grupo se interessavam principalmente "[...] em lógica, álgebra abstrata, fundamentos da matemática, metodologia da ciência e ensino da matemática. Em certo sentido, a origem da pesquisa em lógica no Brasil pode ser remontada às atividades desse grupo"6.

Contudo, Newton da Costa percebeu que o grupo que coordenava não conseguiria realizar em Curitiba o mesmo feito realizado na Polônia, pois estavam mais direcionados a solucionar problemas de ordem fundamental nas ciências. Tendo em vista essas divergências e aquelas de ordem política que não via futuro em Curitiba e na UFPR de pesquisas originais e relevantes, da Costa se mudou para São Paulo em 1968, desenvolvendo nesse local, precisamente em Campinas - SP, um ambiente profícuo para o trabalho matemático em lógica e em fundamentos (MICALI, 2011; DA COSTA; SANT'ANNA, 2016). A mudança de Newton da Costa para Campinas-SP, refletiu nos planos profissionais e científicos de Ayda como veremos.

\section{Ayda e sua trajetória profissional e científica na Unicamp}

Até junho de 1968, Ayda Ignez Arruda permaneceu no quadro de professores da Universidade Federal do Paraná, mudando-se para o estado de São Paulo nesse mesmo ano. Em entrevista concedida a Cavalari (2007), a professora Itala D'Ottaviano afirmou que ao ser contratado pela Unicamp em 1968, o professor Newton da Costa indicou Ayda, que foi contratada como Professora Titular na área de Lógica e Fundamentos da Matemática, do recém-criado Instituto de Matemática, Estatística e Ciência da Computação (IMECC) Unicamp. Isso pode ser comprovado no excerto de da Costa e Alcantara (1985, p. 1, tradução nossa) a seguir: “[...] Em 1968 foi convidada a lecionar no Instituto de Matemática, Estatística e Ciências da Computação da Universidade Estadual de Campinas como Professora Titular de Matemática ${ }^{7}$, referindo-se a Ayda. Nesse período era comum o convite, por meio de indicação de assistentes de professores para assumir cadeiras nas universidades.

Conforme Cavalari (2007) vários docentes nesse período foram contratados como professores titulares, visto que

"Com a institucionalização da universidade, tornou-se necessária aprovação em concurso para obtenção do título de Professor Titular e, para inscrição neste concurso era necessário ser Professor Adjunto (título também obtido mediante aprovação em concurso). No entanto, a UNICAMP concedeu aos professores contratados como Titulares, o direito de permanecerem com este titulo." (CAVALARI, 2007, p. 111)

\footnotetext{
6 "in logic, abstract algebra, foundations of mathematics, methodology of science and the teaching of mathe- matics. In a certain sense, the origin of research in logic in Brazil may be traced back to the activities of this group".

7 "In 1968 she was invited to teach in the Institute of Mathematics, Statistics and Computation Sciences of the State Univeristy of Campinas as Full Professor of Mathematics".
} 
Desse modo, Ayda havia recebido o direito de permanecer como professora titular sem a prestação de concurso para o cargo, porém, conforme relato da professora Itala D’Ottaviano a Cavalari (2007) nossa personagem pretendia ser professora titular concursada $^{8}$.

Com sua ida para Campinas-SP, sob a liderança do professor Newton da Costa, formou-se por volta de 1968 um grupo de jovens lógicos na Unicamp, o conhecido "Grupo de Campinas" - Grupo de Lógica do Departamento de Matemática do Instituto de Matemática, Estatística e Ciência da Computação (IMECC). Esse grupo corroborou eximiamente para promoção da lógica na instituição e para outras localidades a partir desse período. De acordo com D’Ottaviano e Gomes (2011, p. 33), “[...] além de da Costa e Arruda, fizeram parte Antonio Mário Sette, Luiz Paulo de Alcântara, Itala M. Loffredo D’Ottaviano, Marta Sagastume de Galego, Roberto Cignoli, Walter Carnielli”.

Contudo esse grupo só teve impulso a partir da criação do Centro de Lógica, Epistemologia e História da Ciência - CLE que veio a acontecer em 1977 (CLE, s/d). O responsável pela criação do CLE foi o professor Zeferino Vaz, na época Reitor da Unicamp, e a idealização e organização pelo professor Oswaldo Porchat de Assis Pereira da Silva. Influenciado pelo período em que estudou no Group in Logic and the Methology of Science da Universidade da Califórnia, Berkeley em 1969 e 1970, Oswaldo Porchat de Assis Pereira da Silva se motivou a constituir na Universidade Estadual de Campinas um centro nos padrões do grupo que estudara. Ayda também foi membro fundador daquele centro (D’OTTAVIANO; GOMES, 2011a; GOMES; D’OTTAVIANO, 2017; CAVALARI, 2013).

De outra parte, no período inicial que Ayda chegou ao estado de São Paulo, percebemos que seu ingresso como professora da Unicamp possibilitou que ela começasse a fazer intercâmbios profissionais e acadêmicos em outros países. Conforme da Costa e Alcantara (1988), Arruda visitou várias instituições em distintas localidades como professora visitante, bolsista ou conferencista.

"Entre essas instituições estavam as Universidades de Bahía Blanca (Argentina), Buenos Aires, Clermont-Ferrand, Lyon, Varsóvia, Torún, Gent e São Paulo, a Universidade Católica do Chile, a Academia de Ciências da Bulgária e a Academia de Ciências da Polônia9." (DA COSTA; ALCANTARA, 1988, p. 2, tradução nossa)

Em seu curriculum vitae (ARRUDA, 1983) consta que em 1968 foi bolsista da Fundação de Amparo à Pesquisa do Estado de São Paulo (FAPESP) para estagiar nas universidades de Buenos Aires, Bahía Blanca e Córdoba na Argentina. No período que vai de julho a agosto de 1968, Ayda participou de seminários com os professores Antonio

\footnotetext{
${ }^{8}$ Ayda prestou concurso e foi aprovada no cargo de professora Adjunta em 1981. Sua intenção era ser professora Assistente concursada, porém, sua prematura morte a impediu.

9 "Among such institutions were the Universities of Bahía Blanca (Argentina), Buenos Aires, Clermont-Ferrand, Lyon, Warsaw, Torún, Gent and São Paulo, the Catholic University of Chile, The Bulgarian Academy Sciences and The Polish Academy of Sciences".
} 
Monteiro (Universidade Nacional del Sur, Bahia Blanca), Luiz E. Sanchiz (Universidade de Buenos Aires) e Andrés R. Raggio (Universidade de Córdoba) (Processo (n. 26), 1968)

Entre novembro de 1969 e maio de 1970, foi prestigiada com mais uma bolsa da FAPESP para estagiar na Universidade de Clermont-Ferrand e Lyon, França. O convite de estágio foi feito pelo professor Marcel Guillaume, aquele que havia passado uma temporada no Brasil no verão de 1964-1965, estudando com ela e o professor Newton da Costa em Curitiba. Nessa viagem, Ayda trabalhou na teoria dos sistemas formais inconsistentes e visitou as Universidades de Paris, Lyon, Roma, Espanha e Portugal (Processo (n. 26), 1968). Ayda recebeu da Unicamp auxílio para estagiar em Universidades da França, Alemanha Ocidental e Polônia no ano de 1976. Da FAPESP, recebeu a passagem para viagem de estudos ao Chile, em janeiro de 1977, onde participou de seminários, proferiu palestras e foi professora visitante na Universidade Católica do Chile.

Enquanto estava na Universidade de Clermont-Ferrand, sob orientação do professor Marcel Guillaume, em 1969-1970, Ayda frequentou os Seminaires de Logique et de Logique Algèbrique e um curso sobre Álgebra ministrado por Guillaume, além de ser conferencista em duas oportunidades nessa universidade e outras duas na Universidade de Lyon, produzindo durante o período que esteve na França, quatro trabalhos acadêmicos: - Sur les systémes $N F_{i}$ de da Costa, nota apresentada à Academia de Ciências de Paris em 13 de abril de 1970; - Sur le système $N F_{w}$, nota apresentada à Academia de Ciências de Paris em 13 de abril de 1970; - La mathématique classique dans le système $\mathrm{NF}_{\mathrm{w}}$, a ser apresentada à Academia de Ciências de Paris; - Sur les systèmes formels inconsistants, a aparecer nos Anais da Universidade de Clermont-Ferrand.

Destacamos a conclusão de Ayda sobre essa viagem nos mais diferentes aspectos tratados por ela, a partir do seu Relatório de Viagem:

"Nossa viagem foi interessante sobre todos os pontos de vista, principalmente o profissional. Em particular convém lembrar aqui alguns dos pontos positivos da mesma: a) estivemos em contacto com matemáticos de grande projeção o que nos deu a possibilidade de tomamos conhecimento do que se faz em alguns excelentes centros de investigação; b) mediante conferências e palestras de caráter particular difundimos o que atualmente faz o grupo de lógica da Universidade Estadual de Campinas, c) as condições de viagem permitiram o desenvolvimento de numerosas de nossas idéias, o que certamente influenciará a nossa atividade científica futura. Em síntese, a nossa viagem contribuindo para nossa evolução científica e profissional trará certamente benefícios para a Universidade Estadual de Campinas e para o desenvolvimento da matemática brasileira." (Processo (n. 26), 1968, 119)

Diante do que narramos, percebemos que a década de 1970 foi frutífera para Ayda em termos de seus estudos, participações e organizações de eventos da lógica matemática, recebendo diversos auxílios de diferentes instituições e de outros países, como da Universidade Católica de Santiago, Chile, no ano de 1970, para participar do I Simpósio Latino Americano de Lógica - SLALM, evento que reuniu muitos brasileiros, como 
convidados e palestrantes (ARRUDA, 1983; D’OTTAVIANO; GOMES, 2011). Recebeu auxílio da Unicamp para participar da III Conferência Latino Americano de Educação Matemática, Bahía Blanca, Argentina, em 1972. Da FAPESP para participar do II Simpósio Latino-Americano de Lógica Matemática - SLALM, Brasília, em julho de 1972, evento que reuniu diversos lógicos como: Rolando Chuaqui (Chile), Roberto Cignoli (Argentina), Maximiliano Dickmann (França), Marcel Guillaume (França), Luis Monteiro (Argentina), Abraham Robinson (EUA), Patrick Suppes (EUA), a própria Ayda e Newton da Costa (GOMES; D'OTTAVIANO, 2011).

Nesse meio tempo de viagens e eventos, no Instituto de Matemática da Unicamp foi membro de bancas julgadoras da tese de mestrado do professor Antonio Mário Sette em 19 de abril de 1971; membro das bancas de exame de qualificação de mestrado e língua estrangeira dos professores Benjamin Bordin, Otilia Paques, Itala D’Ottaviano e Paltonio Fraga em novembro e dezembro de 1971; membro da banca julgadora da tese de mestrado da professora Itala D'Ottaviano em 15 de março de 1974; membro da comissão examinadora para a seleção de candidatos à docência no Departamento de Matemática e Física da Faculdade de Filosofia, Ciências e Letras de Araraquara em 27 de fevereiro de 1974 (Processo (n. 26), 1968). Até 1982 Ayda também orientou duas pesquisas de Mestrado, das alunas Clayde R. M. Dos Santos ${ }^{10}$ e Regina M. Moreno ${ }^{11}$, e também participou de vários eventos nacionais e internacionais, foi membro das bancas de doutorado de Itala M. L. D’Ottaviano e Walter Carnielli. Também foi professora visitante de várias instituições.

Orientou também a bolsa de iniciação científica pela FAPESP de Maria Lúcia Campos e Ana Lúcia Junqueira em 1973. E entre 1972-1974 foi coordenadora dos projetos sobre ensino de Matemática do IMECC (ARRUDA, 1983), cujos nomes apresentamos a saber: "Integração Escola de $2^{\circ}$ grau-Universidade", projeto em parceria com a secretaria de educação de Campinas-SP; "Projeto: Prática de Ensino da Matemática", 1972-1973; "Projeto: Curso de Aperfeiçoamento para professores secundários", desde agosto de $1974 \mathrm{e}$ "Projeto: Palestras em escolas de $1^{\circ}$ e $2^{\circ}$ graus da região de Campinas sobre o curso de Matemática" (Processo (n. 26), 1968).

Face a isso, destacamos o trabalho de inserção de jovens estudantes que Ayda perpetrava na Unicamp para aprofundamento da lógica matemática. Segundo Walter Carnielli citado por Gomes e D'Ottaviano (2017, p 668) quando este era estudante de matemática na Unicamp, por volta de 1975, a professora Ayda "[...] convidou um pequeno grupo de estudantes para se aprofundar extra-curricularmente em lógica", neste grupo estava a professora Itala. Ainda, segundo Carnielli, a influência da professora Ayda Arruda foi decisiva em sua carreira acadêmica, assim como dos professores Newton da Costa, Andrés Raggio e Rolando Chuaqui.

De outra parte, se referindo a organização de eventos e participações de Ayda Arruda, temos que em março de 1975, Alfred Tarski (um dos mais reconhecidos lógicos de

\footnotetext{
${ }^{10}$ SANTOS, Clayde Regina Mendes dos. Sobre o sistema NFw. 1980. 116 f. Dissertação (mestrado) - Universidade Estadual de Campinas, Instituto de Matematica, Estatistica e Computação Científica, Campinas, SP. Disponível em: <http://www.repositorio.unicamp.br/handle/REPOSIP/306761>. Acesso em: 11 jul. 2021.

${ }^{11}$ FONTE, Regina A. Munhoz da. N. A. Vasiliev e a lógica não-classica. 1983. 88f. Dissertação (mestrado) Universidade Estadual de Campinas, Instituto de Filosofia e Ciencias Humanas, Campinas, SP. Disponível em: <http://www.repositorio.unicamp.br/handle/REPOSIP/281713>. Acesso em: 11 jul. 2021.
} 
todos os tempos) junto a Rolando Chuaqui (que já havia sido professor do instituto) visitou o Instituto de Matemática, Estatística e Ciência da Computação (IMECC) da Unicamp. Ayda Arruda e Newton da Costa aproveitaram a visita de Tarski à América do Sul e o convidaram para visitar o instituto:

"Tarski passou alguns dias em Campinas em apresentou duas conferências sobre álgebra relacional, as quais foram gravadas de forma pioneira em vídeo e preservadas no acervo dos Arquivos Históricos do Centro de Lógica, Epistemologia e História da Ciência (CLE) da Unicamp." (SUGUITANI; VIANA; D’OTTAVIANO, 2016, p. 8)

Na ocasião de sua visita, Ayda organizou um Simpósio de Lógica Matemática no IMECC, entre os dias 3 e 5 de março, na qual Tarski apresentou duas conferências sobre álgebra e questões em aberto na época. O evento contou “[ [...] com a participação de lógicos e alunos da Unicamp, USP, e da Universidade Federal de Pernambuco (UFPE), cujos anais foram publicados por Arruda com a colaboração de Itala M. Loffredo D'Ottaviano". Esse foi o primeiro evento sobre lógica matemática organizado por Ayda (D’OTTAVIANO; GOMES, 2011a).

Suguitani, Viana e D’Ottaviano (2016) organizaram um livro bilíngue, com a transcrição das conferências na Unicamp em 1975 de Alfred Tarski, além de reproduzirem no apêndice do livro as atas do simpósio, que segundo eles foram editadas por Ayda Arruda (inclusive era ela quem desenhava os símbolos lógicos, pois na época não existiam máquinas datilográficas com todos os símbolos) e pioneiramente publicadas pelo IMECC.

O segundo evento organizado por Ayda, como presidente da comissão, aconteceu no ano seguinte. Visto que com o aumento de adeptos a Lógica no Brasil, foi decidido que o III Simpósio Latino-Americano de Lógica Matemática - SLALM se realizaria no IMECC em Campinas entre os dias 12 e 16 de julho de 1976 com apoio da Association for Symbolic Logic:

“[...] precedido por todo um semestre de cursos e seminários para estudantes de pós-graduação. Constou de 3 sessões: Teoria de Modelos, Lógicas Não-Clássicas e Computabilidade. A organização de Ayda Arruda foi primorosa e o sucesso do simpósio grande, com a participação ativa de muitos brasileiros e jovens lógicos latino-americanos, entre eles Xavier Caicedo (Colômbia), Irene Mikemberg e Manuel C. Corrada (Chile). Diversos lógicos importantes participaram como conferencistas convidados, entre eles F. G. Asenjo (EUA), M. Benda (EUA), R. Chuaqui (Chile), R. Fraissé (França), J. Kotas (Polônia), M. Krasner (França), E.G.K. López-Escobar (EUA), C. Pinter (EUA), R. Routley (Austrália), J. $R$. Shoenfield (EUA) e R. Solovay (EUA). Numa das sessões desse simpósio, deu-se um importante evento relativo à lógica paraconsistente: a proposição, pelo filósofo peruano Francisco Miró Quesada, do termo 'paraconsistente' para nomear as lógicas que possam ser subjacentes a 
teorias inconsistentes e não-triviais ${ }^{12}$ " (D'OTTAVIANO; GOMES, 2011b, p. 12-13, grifo nosso).

Segundo D’Ottaviano e Gomes (2020, no prelo), foi nesse simpósio organizado por Ayda Arruda que Francisco Miró Quesada Cantuarias proferiu a palestra sobre "Lógicas heterodoxas e o problema da unidade de lógica" no dia 15 de julho de 1976. Nessa ocasião, tornou pública e sugestiva os termos 'lógica paraconsistente' e 'paraconsistência'. De acordo com esses autores, dentre os reconhecidos lógicos de outros países que participaram deste evento, diversos jovens lógicos que testemunharam aquele momento são reconhecidos na lógica latino-americana atualmente, como a professora Itala D'Ottaviano, que naquela época era estudante de doutorado e acompanhou este evento, inclusive a palestra de Miró Quesada com a questão do termo paraconsistente.

Esse evento foi importante para a comunidade da lógica matemática no Brasil, por ser o primeiro evento continental da área no país, marcando a liderança brasileira no que se relaciona a lógica matemática na América Latina.

Segundo Aliseda e Manzano (2015) os anais desses simpósios ocorridos no Brasil, precisamente o segundo e o terceiro (1972 e 1976), foram publicados pela editora North Holland em 1977, na série "Studies in Logic and the Foundations of Mathematics", editados por Ayda Ignez Arruda, Newton Carneiro Affonso da Costa e Rolando Chuaqui, com o título "Nonclassical logics, model theory and computability". Esses simpósios aconteciam(em) a cada dois ou três anos e corroboram no desenvolvimento dos estudos da lógica na América Latina.

Outro evento que foi organizado por Ayda foi o I Encontro Brasileiro de Lógica EBL, ocorrido em Campinas, de 4 a 6 de julho de 1977, evento sob responsabilidade do CLE, também criado em 1977. Arruda foi presidente do Comitê Organizador desse encontro (Processo (n. 26), 1968). Segundo D’Ottaviano e Gomes (2011a, p. 17, grifo do autor) o objetivo dos encontros era reunir lógicos brasileiros e estimular a pesquisa nacional nessa área. Esse primeiro encontro contou com a participação de pessoas provenientes de dez universidades brasileiras. Os anais da conferência "[...] foram publicados em 1978, pela Marcel Dekker, sob o título Mathematical Logic - Proceedings of the First Brazilian Logic Conference, editados por A. I. Arruda, N. C. A. da Costa e R. Chuaqui".

Ayda também foi presidente do Comitê Organizador do II Encontro Brasileiro de Lógica, na Unicamp, ocorrido entre 3 a 7 de julho de 1978. Esse foi o segundo de vários encontros que o CLE organizava anualmente na Unicamp, seu comitê organizador foi composto pelos seguintes professores da Universidade: Ayda I. Arruda, Elias H. Alves,

\footnotetext{
12 "Preceded by an entire semester of courses and seminars for graduate students. The Symposium consisted of three sessions: model theory, nonclassical logic, and computability. The event, excellently organized by Ayda Arruda, was a great success. Many young logicians from Brazil and other Latin American countries attended, among them Xavier Caicedo (Colombia), Irene Mikenberg (Chile), and Manuel C. Corrada (Chile). Several important logicians participated as guest lecturers, including F.G. Asenjo (USA), M. Benda (USA), R. Chuaqui (Chile), R. Fraissé (France), J. Kotas (Poland), M. Krasner (France), E.G.K. López-Escobar (USA), C. Pinter (USA), R. Routley (Australia), J. R. Shoenfield (USA), and R. Solovay (USA). At one of the sessions of the symposium there occurred an important event relative to paraconsistent logic: the proposal by Francisco Miró-Quesada of the term 'paraconsistent' for referring to logics that can underlie non-trivial inconsistent theories".
} 
Newton C. A. da Costa e Oswaldo Porchat Pereira. Neste evento, cursos foram ministrados, além de "[...] conferências e comunicações, estando presentes os seguintes conferencistas: prof. Oswaldo Chateaubriand (PUC, Rio), Roberto Cignoli (Unicamp), Carlos Pinter (USA), Luiz Paulo de Alcântara (Unicamp) e Leslaw Szczerba (Polônia)" (CIÊNCIA E CULTURA (SP), 1978, p. 1148).

A par disso, podemos enfatizar uma fala da professora Itala D'Ottaviano concedida a pesquisadora Cavalari (2007) quanto ao papel que Ayda representou para a divulgação das pesquisas sobre lógica matemática, visto que "[...] foi graças ao trabalho da Ayda que os Encontros Brasileiros de Lógica começaram a ter Atas publicadas por editoras internacionais" e que "[...] os simpósios Latino-Americanos e Brasileiros começaram a fazer parte do calendário internacional" (CAVALARI, 2007, p.113, grifo do autor).

Verificamos que Ayda foi professora visitante no Instituto de Matemática da Universidade Católica do Chile em janeiro de 1978 e, posteriormente, de outubro até dezembro do mesmo ano. Ayda foi membro do Comitê Organizador do IV Simpósio LatinoAmericano de Lógica Matemática, que se realizou naquela instituição em dezembro daquele ano; também foi membro do Comitê Organizador do V Simpósio Latino-Americano de Lógica Matemática realizado em Bogotá, na Colômbia em 1981 (Processo (n. 26), 1968).

As contribuições de Ayda são inquestionáveis, seu comprometimento e sua disposição na faceta pesquisadora e profissional são compromissos que nossa personagem assumiu quase uma década antes desses eventos. Devemos ressaltar que no Plano de Pesquisa escrito por Ayda em 24 de maio de 1968, para justificar a mudança de regime de turno parcial para turno de dedicação integral, enquanto professora do IMEEC, ela se propôs a colaborar com a linha de pesquisa daquele instituto e a fomentar a pesquisa.

"Finalmente achamos oportuno lembrar que uma das facetas mais importantes de nossa atividade científica constituir-se-á na realização de seminários de pesquisas, e de cursos de pós-graduação; êstes têm a finalidade de orientar e conduzir os elementos jovens do Instituto de Matemática da Universidade de Campinas, ou de outros centros, relativamente a pesquisa e a carreira matemática" (Processo (n. 26), 1968, fl. 70).

Baseadas nessa proposição, verificamos que Ayda se colocou a cumprir o que havia proposto em seu plano de pesquisa até seu prematuro falecimento, desde a organização de eventos e seminários, até a orientação e condução de jovens interessados a estudar lógica matemática. Sua faceta profissional, suscitava reconhecimento, o que possibilitava que seu contrato enquanto professora da Unicamp fosse prorrogado mediante seu desempenho enquanto professora e pesquisadora por diversas vezes até maio de 1979, conforme pudemos perceber no Processo n. 26 (1968), passando a ser indeterminado a partir de junho de 1979, devendo Ayda a cada dois anos apresentar um relatório de suas atividades ao conselho diretor.

No período de 1979 a 1981, conforme seu relatório de atividades (Processo (n. 26), 1968), Ayda desempenhou atividades de gestão: de 16 de junho de 1979 a 15 de abril de 1980 foi Chefe do Departamento de Matemática do IMECC, foi membro da Congregação do 
IMECC e membro do Conselho do Departamento de Matemática do IMECC (entre junho de 1977 a junho de 1979), foi a primeira Diretora eleita do IMECC, a partir de 16 de abril de 1980, substituindo o professor Ubiratan D'Ambrosio, dirigente desde 1972. Conforme relato do professor João Frederico Meyer para Roldan (2014) foi fantástico para a Matemática a indicação e eleição de uma mulher como diretora. Ayda se manteve no cargo até o seu falecimento em 1983.

Também é importante destacar que Ayda foi uma das sócias fundadoras na Sociedade Brasileira de Lógica (SBL), entidade criada em 14 de fevereiro de 1979, em reunião realizada no IMECC. Contudo, Gomes (2013, p. 485) destaca que foi em 1977 que da Costa, "[...] apoiado por Ayda Ignez Arruda, concebeu a criação da Sociedade Brasileira de Lógica, tendo sido seu primeiro Presidente". Segundo o Processo (n. 26, 1968) Ayda Ignez Arruda entre os anos 1979-1980 foi vice-presidente desta sociedade e entre os anos 19811982 foi presidente da SBL.

Em 1981 Ayda como diretora do IMECC, solicitou a reitoria abertura de concurso para a função de professora adjunta na área de Fundamentos e História da Matemática, do Departamento de Matemática do IMECC para ela mesma. Não houve inscrições de outras pessoas e o concurso tinha o objetivo de julgar os títulos dos candidatos. Participaram da banca no dia 24 de agosto de 1981 os professores doutores Alexandre Rodrigues, Edison Farah, Constantino Menezes de Barros, Newton Carneiro Affonso da Costa e Roberto Leonardo Oscar Cignoli, sendo aprovada com média 9,8 (Fundo AIA/ Arquivo Históricos/ CLE-Unicamp). Ayda se manteve no cargo de Diretora do IMECC, de abril de 1980 até o seu falecimento em 1983. Nesse período, foi exonerada devido ao episódio de intervenção da UNICAMP em 1981 e retornou ao cargo após liminar judicial.

Ayda era solteira e não tinha filhos, faleceu em virtude de um câncer, na cidade de Campinas-SP, no dia 13 de outubro de 1983. Seu legado para a lógica matemática, sobretudo a história da Lógica paraconsistente no Brasil é notável. Após seu falecimento, a fim de homenageá-la e, de certo modo, rememorá-la, alguns trabalhos foram escritos em sua memória, a saber: uma breve biografia de sua vida escrita por Roberto Cignoli (1984) e publicada na Studia Logical3; um artigo que caracteriza seu trabalho científico escrito por Newton da Costa e Luiz Paulo de Alcântara (1985) publicado nos anais do VII Simpósio Latino-Americano de Lógica Matemática - SLALM, evento que aconteceu em Campinas SP em 1985, que também a homenageou.

\section{Algumas considerações}

Neste artigo, nos colocamos a caracterizar historicamente a trajetória profissional e científica de Ayda Ignez Arruda na Universidade Estadual de Campinas (Unicamp), nos anos 1968 a 1983. Tendo feito isto, é notório advertir que Ayda até o seu prematuro falecimento, vivia plenamente em atividades científicas para a Unicamp, cumprindo fielmente seu Plano de

\footnotetext{
${ }^{13}$ Studia Logica publishes original papers on various logical systems, which utilize methods of contemporary formal logic, such as those of algebra, model theory, proof theory and others. Disponível em: < https://www.springer.com/journal/11225 >. Acesso em: 29 jun. 2021.
} 
Pesquisa de seu ingresso como professora desta instituição e no Instituto de Matemática, Estatística e Computação Científica (IMECC).

Sua representatividade se expressava nos cargos que assumia, seja como a primeira mulher eleita como Diretora do IMECC, na criação do CLE (1977) ou como uma das cofundadoras e também vice-presidente (1979-1980) e presidente (1981-1982) da Sociedade Brasileira de Lógica, como responsável por seminários de estudos sobre Lógica na Unicamp, como editora de anais de eventos da área e divulgação internacional desta, como colaboradora e incentivadora de eventos na área, como já apontamos. Seu carisma e entusiasmo são características presentes nos discursos de muitos pesquisadores e amigos(as), que a tinham com muito carinho, e também indicam o seu reconhecimento profissional e científico.

Na sequência, apresentamos uma linha do tempo que construímos, com o intuito de expressar alguns dos principais fatos da vida/carreira acadêmica de Ayda Ignez Arruda. 


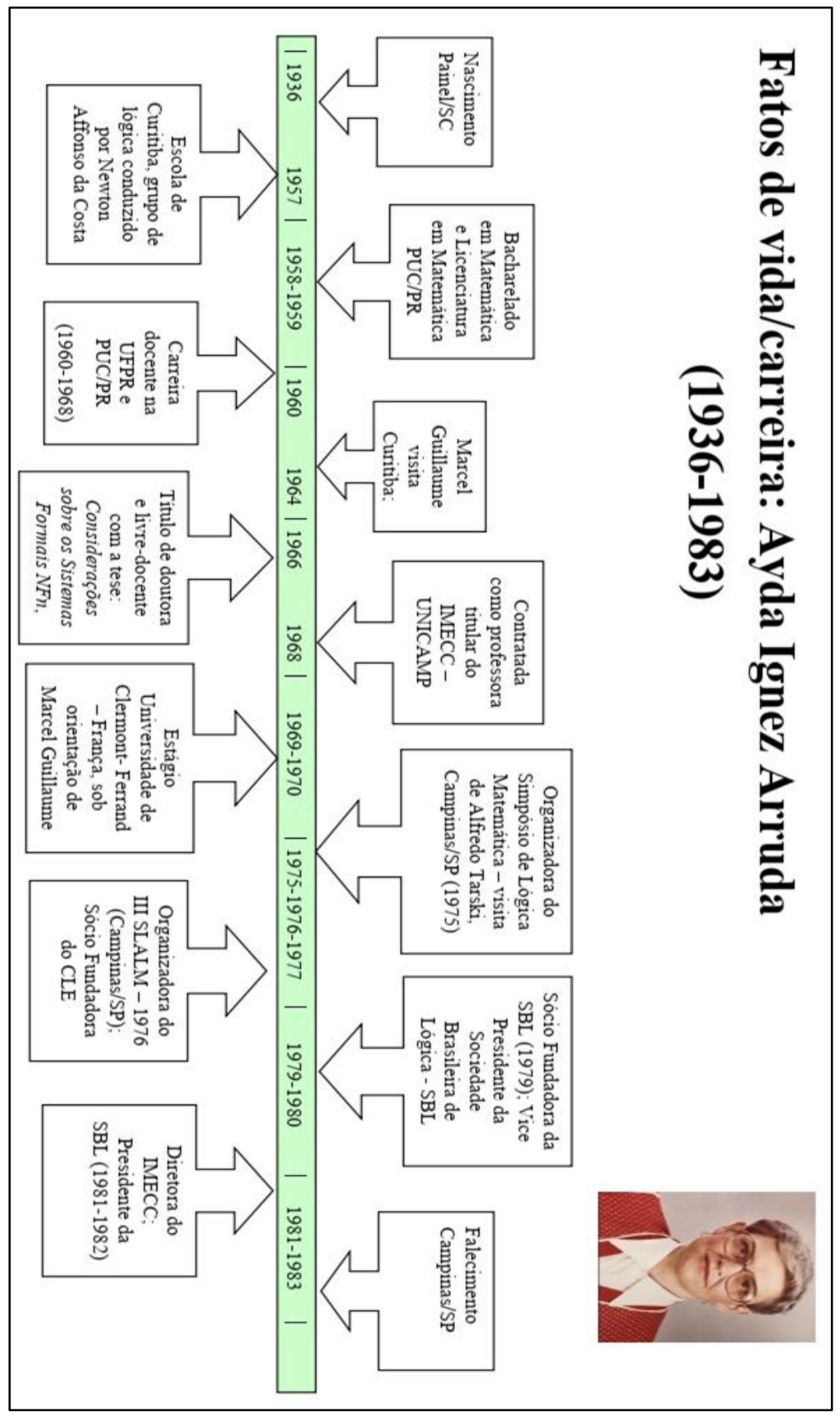




\section{Bibliografia}

ALISEDA, A.; MANZANO, M. 2015. Apuntes para una historia de la lógica em Iberoamérica en el siglo XX. Filosofía iberoamericana del siglo, p. 101.

AQUINO, E. M. L. 2006. Gênero e ciência no Brasil: contribuições para pensar a ação política na busca da equidade. In: BRASIL. Presidência da República. Secretaria Especial de Políticas para as Mulheres. Encontro Nacional de Núcleos em Grupos de Pesquisas. Brasília: Secretaria Especial de Políticas para as Mulheres.

ARAUJO NETO, A. P. 2019. Remy Freire e suas contribuições para a Matemática e Educação Matemática no Paraná. Tese (doutorado) - Universidade Estadual de Maringá, Centro de Ciências Exatas, Programa de Pós-Graduação em Educação para a Ciência e a Matemática.

ARRUDA, A. I. 1983. Curriculum vitae. Datilografado, assinado. Campinas, 14p. (FNCAC, DP, Cx. 24, Ps. 1, 3).

BADIN, M. G. 2006. Um olhar sobre as contribuições do professor Nelson Onuchic para o desenvolvimento da matemática no Brasil. 154 f. Dissertação (mestrado) - Universidade Estadual Paulista, Instituto de Geociências e Ciências Exatas.

BONFIM, S. H. 2013. Theodoro Augusto Ramos: um estudo comentado de sua tese de doutoramento. 124 f. Tese (doutorado) - Universidade Estadual Paulista Júlio de Mesquita Filho, Instituto de Geociências e Ciências Exatas.

BORGES, V. P. 2008. Fontes biográficas: grandezas e misérias da biografia. In: PINSKY, C. B. (org.). Fontes Históricas. São Paulo: Contexto, pp. 203-233.

CALABRIA, A. R. 2015. Francisco Antonio Lacaz Netto (1911-1991): um estudo biográfico. 205 f. Tese - (doutorado) - Universidade Estadual Paulista, Instituto de Geociências e Ciências Exatas.

CAVALARI, M. F. 2007. A matemática é feminina? Um estudo histórico da presença da mulher em institutos de pesquisa em matemática do estado de São Paulo. 147 f. Dissertação (mestrado) - Universidade Estadual Paulista, Instituto de Geociências e Ciências Exatas.

CAVALARI, M. F. 2012. As contribuições de Chaim Samuel Hönig para o desenvolvimento da matemática brasileira. 202 f. Tese - (doutorado) - Universidade Estadual Paulista, Instituto de Geociências e Ciências Exatas.

CAVALARI, M. F. 2013. Mulheres Pioneiras na Matemática no Brasil. Revista do Professor de Matemática, v. 30, pp. 05-09.

RBHM, Vol. 21, n 41, pp. 25-44, 2021 
CIGNOLI, R. 1984. Ayda Ignez Arruda (1936-1983). Studia Logica, v. 43, n. 1-2.

CLE. Centro de lógica, epistemologia e história da ciência - Unicamp. Ayda Ignez Arruda. s/d. Disponível em: <https://www.cle.unicamp.br/index.php/ayda-ignez-arruda>. Acesso em: 24 maio. 2021.

COBRA, T. T. L. 2014. Carlos Benjamin de Lyra e a topologia algébrica no Brasil. 125 f. Tese - (doutorado) - Universidade Estadual Paulista, Instituto de Geociências e Ciências Exatas.

COUSIN, A. O. A. 2007. A Sociedade Paranaense de Matemática sob um olhar da Educação Matemática. 254 f. Tese (Doutorado) - Curso de Setor de Educação, Programa de Pósgraduação em Educação, Universidade Federal do Paraná, Curitiba.

DELORY-MOMBERGER, C. 2011. Fundamentos epistemológicos da pesquisa: biográfica em educação. Educação em Revista, v. 27, n. 1, pp. 333-346.

DA COSTA, N. C. A. 1986. On paraconsistent set theory. Logique et Analyse, v. 29, n. 115, pp. 361-371.

DA COSTA, N. C. A. 2016. Avaliando Avaliadores. In: SANTOS, C. H.; MIYAÒKA, F. K.; BARREDA, M. J. C. Sete décadas do curso de Matemática da UFPR. Curitiba-PR: Ed. UFPR, pp. 96-104.

DA COSTA, N. C. A.; AlCÂNTARA, L. P. 1985. The Scientific Work of AI Arruda. Universidade Estadual de Campinas. Instituto de Matemática, Estatística e Ciência da Computação, 1985. Disponível em: <https://bookstore.ams.org/conm-69/14>. Acesso em: 02 abr. 2021.

D'OTTAVIANO, I. M. L.; CARNIELLI, W. A.; ALVES, E. H. 1996. The Centre for Logic in Campinas and the development of logic in Brazil. Logique et Analyse, v. 39, n. 153/154, pp. $15-29$.

D'OTTAVIANO, I. M. L.; GOMES, E.L. (2011a). Considerações sobre o desenvolvimento da lógica no Brasil. CLE e-Prints (Online), v. 11, pp. 1-50, 2011a.

D’OTTAVIANO, I. M. L.; GOMES, E. L. (2011b) On the development of logic in Brazil I: the early logic studies and the path to contemporary logic. Revista Brasileira de História da Matemática, v. 11, n. 22, pp. 03-28.

D’OTTAVIANO, I. M. L.; GOMES, E. L. 2020. Baptizing Paraconsistent Logics: the unique touch of Miró Quesada. South American Journal of Logic. v. 6, n. 2, pp. 1-21. No prelo. 
DUARTE, P. C. X. 2014. Candido Lima da Silva Dias: da Politécnica aos primórdios da FFCL da USP. 2014. 140f. Tese - (doutorado) - Universidade Estadual Paulista, Instituto de Geociências e Ciências Exatas.

ESTADO DE SANTA CATHARINA, VOL. IV. Munícipios. 1930. Disponível em: < http://memoria.bn.br/DocReader/Hotpage/HotpageBN.aspx?bib=313394\&pagfis=107361\& url=http://memoria.bn.br/docreader\#>. Acesso em: 25 jun. 2021.

Fundo Ayda Ignez Arruda/ Arquivos Históricos/ CLE-Unicamp.

GOMES, E. L. 2005. O desenvolvimento da lógica no Brasil: da herança ibero-portuguesa aos primórdios do século XIX. Revista Eletrônica Informação e Cognição, v. 4, n. 1.

GOMES, E. L.; D'OTTAVIANO, I. M. L. 2017. Para além das Colunas de Hércules, uma história da paraconsistência: de Heráclito a Newton da Costa. Campinas, SP: Editora da Unicamp.

IMECC50. 2018. História. Disponível em:〈http://www.imecc50.ime.unicamp.br/historia〉. Acesso em: 23 maio. 2021.

JENKINS, K. 2007. A história repensada. Tradução de Mário Vilela, São Paulo, Contexto.

LEMOS, G. C. 2013. Willie Alfredo Maurer: vida, obras e contribuições para o ensino da Matemática no Brasil. 2013. 172 f. Tese - (doutorado) - Universidade Estadual Paulista, Instituto de Geociências e Ciências Exatas.

LOPES, A. C. C. 2014. A presença feminina no corpo docente dos cursos de engenharia das instituições precursoras da Universidade Federal de Itajubá. 103 f. Mestrado Profissional em ensino de ciências. Universidade Federal de Itajubá, Itajubá.

MICALI, A. 2011. Newton da Costa and the school of Curitiba. Manuscrito, v. 34, n. 1, p. $21-50$.

PEREIRA, P. C. 2010. A Educadora Maria Laura: contribuições para a constituição da Educação Matemática no Brasil. 2010. 155f. Tese (doutorado) - Pontifícia Universidade Católica de São Paulo, São Paulo.

Processo (n. 26). 1968. Processo de Vida Funcional de Ayda Ignez Arruda. Arquivo Central/Siarq da Unicamp, Campinas, 607p.

ROLDAN, R. 2014. Intervenção Unicamp o filme. Youtube. Disponível em: <https://www.youtube.com/watch?v=BSPTv4a7ITo\&t=218s>. Acesso em: 01 out. 2021.

RBHM, Vol. 21, n ${ }^{\circ} 41$, pp. 25-44, 2021 
SAITO, F. 2015. História da matemática e suas (re)construções contextuais. São Paulo: Livraria da Física/SBHMat.

SANTOS, A. A. 2010. Elza Furtado Gomide e a participação feminina no desenvolvimento da matemática brasileira no século XXI. Dissertação de Mestrado. Pontifícia Universidade Católica de São Paulo, São Paulo.

SANTOS, C. H.; MIYAÒKA, F. K.; BARREDA, M. J. C. 2016. Sete décadas do curso de Matemática da UFPR. Curitiba-PR, Ed. UFPR.

SILVA, C. P. 2001. Desenvolvimento da matemática no Paraná: um estudo do caso Universidade Federal do Paraná. Llull: Revista de la Sociedad Española de Historia de las Ciencias y de las Técnicas, v. 24, n. 49, p. 183-200.

SOUTO, R. M. A. 2006. Mario Tourasse Teixeira: o homem, o educador, o matemático. 151 f. Tese (doutorado) - Universidade Estadual Paulista, Instituto de Geociências e Ciências Exatas.

SUGUITANI, L.; VIANA, J. O.; D’OTTAVIANO, I. M. L. (orgs.). 2016. Conferências na Unicamp em 1975 - Alfred Tarski = Lectures at Unicamp in 1975-Alfred Tarski. Campinas, SP, Editora da Unicamp.

TRIVIZOLI, L. M. 2016. Um panorama para a investigação em história da matemática: surgimento, institucionalização, pesquisas e métodos. Revista Paranaense de Educação Matemática, v. 5, n. 8.

Suélen Rita Andrade Machado
Departamento de Matemática - Faculdade de Engenharia e
Inovação Técnico Profissional - FEITEP - Maringá - Bra-
sil
E-mail: sumachado18@gmail.com

\section{Lucieli M. Trivizoli}

Departamento de Matemática - Universidade Estadual de Maringá - UEM - Maringá - Brasil

E-mail: 1mtrivizoli@uem.br 